\title{
Meconium Peritonitis: In Utero Diagnosis of a Rare Clinical Entity and Postnatal Outcome
}

\section{Sarita Agrawal, MD, FICOG, FIAMS, FCGP', Arpana Verma, MS $^{2 *}$, Sarita Rajbhar, MS ${ }^{3}$, Pushpawati Thakur, MD', Loukya Kodumuri, MBBS ${ }^{5}$ and Swati Kumari, MS, FNB ${ }^{6}$}

${ }^{1}$ Professor and Head of Department, Department of Obstetrics and Gynaecology, All India Institute of Medical Sciences, Raipur, Chhattisgarh, India

${ }^{2}$ Senior Resident, Department of Obstetrics and Gynaecology, All India Institute of Medical Sciences, Raipur, Chhattisgarh, India

${ }^{3}$ Assistant Professor, Department of Obstetrics and Gynaecology, All India Institute of Medical Sciences, Raipur, Chhattisgarh, India

${ }^{4}$ Associate Professor, Department of Obstetrics and Gynaecology, All India Institute of Medical Sciences, Raipur, Chhattisgarh, India

${ }^{5}$ Post Graduate Student, Department of Obstetrics and Gynaecology, All India Institute of Medical Sciences, Raipur, Chhattisgarh, India

${ }^{6}$ Sonologist and Fetal Medicine Expert, Shivam Fetomed and Spine Centre, Raipur, Chhattisgarh, India

*Corresponding author: Arpana Verma, MS, Senior Resident, Department of Obstetrics and Gynaecology, All India Institute of Medical Sciences, V.V-18, Parthivi Province, Near Salasar Greens, Sarona, Raipur, Chhattisgarh - 492099, India, Tel: $9741412716 ; 6260334337$

\begin{abstract}
Objective: To present an unusual case of meconium peritonitis diagnosed during prenatal period and its postnatal outcome.

Background: Meconium peritonitis (MP) is a rare cause of non-immune hydrops with reported incidence of 1:35,000 live births. MP is defined as an aseptic localized or generalized peritonitis caused due intrauterine bowel perforation and extravasation of the meconium. Few causes which might result in perforation include lleal atresia, intussusception, Hirschsprung's disease, cystic fibrosis, volvulus, colonic atresia, Meckel diverticulitis and vascular insufficiency. Successful outcome with conservative management has been seen in limited number of cases, however, surgery is imperative when signs and symptoms of intestinal obstruction are present. Favorable outcome have been seen when the condition was detected in utero rather than when the neonatal diagnosis is made.
\end{abstract}

Case: A 32 year, Multigravida was referred to our hospital at 33 weeks 2 days of gestation in view of isolated fetal ascites, diagnosed on antenatal scan at 32 weeks. Antenatal workup done for immune and non-immune hydrops and was found to be negative. One week later, a repeat ultrasound was done which showed moderate fetal ascites with few areas of calcification in the bowel loops and prominent inferior vena cava, there was also associated polyhydramnios. The provisional diagnosis of MP was made. She was given injection dexamethasone for fetal lung maturation with a plan for delivery at 37 completed weeks, however spontaneous labour sets in and a preterm hydropic female baby was delivered at 35 weeks. She needed intubation and ventilator support. Post natal ultrasound showed gross ascites with a giant cyst compressing the inferior vena cava, and minimal bilateral pleural effusion. Hence an emergency laparotomy was performed. Intraoperative finding revealed giant meconium cyst bounded with fibrous tissue, on dissection showed terminal ileal perforation. Drainage of meconium cyst and Double-barrel ileostomy was performed. Postoperatively the respiratory symptoms improved. Overall improvement in the general condition of the baby was seen at one week follow up. Unfortunately the baby's condition started deteriorating by third week of life and she started developing signs and symptoms of septicemia. Inspite of active management the baby could not be saved and succumb to sepsis on fourth week of life.

Citation: Agrawal S, Verma A, Rajbhar S, Thakur P, Kodumuri L, et al. (2020) Meconium Peritonitis: In Utero Diagnosis of a Rare Clinical Entity and Postnatal Outcome. Obstet Gynecol Cases Rev 7:180. doi. org/10.23937/2377-9004/1410180

Accepted: October 21, 2020: Published: October 23, 2020

Copyright: (c) 2020 Agrawal S, et al. This is an open-access article distributed under the terms of the Creative Commons Attribution License, which permits unrestricted use, distribution, and reproduction in any medium, provided the original author and source are credited. 
Conclusion: MP needs to be considered if the fetus has isolated ascites with internal echoes and progresses to hydrops, as it is one of the treatable causes of hydrops fetalis. Perinatal morbidity and mortality rate is about $80 \%$. In-utero diagnosis and timely intervention are associated with favourable perinatal outcome. Neonatal septicemia is the most common cause of postoperative morbidity and mortality with an added risk of prematurity as was in our case.

\section{Keywords}

Hydrops fetalis, Non-immune hydrops, Meconium peritonitis, Meconium pseudocyst

\section{Introduction}

Hydrops fetalis (HF) is the result of an imbalance in the regulation of fluid, leading to an increase in interstitial fluid production or a decrease in lymphatic return. HF can be diagnosed prenatally by ultrasound and defined by the presence of more than two abnormal fluid collections (ascites, pericardial/pleural effusion, skin edema) in the fetus. Non-immune hydrops fetalis (NIHF) comprises the subgroup of cases not caused by red cell alloimmunization [1]. The prevalence of NIHF in the general population is estimated to be 1 in every 2500-3500 neonates and 1 in every 1600-7000 fetuses [2]. Wide variations in reported prevalence are due to difference in definitions, populations, thoroughness of evaluation, and whether late pregnancy terminations were included. Despite extensive investigations, the etiology of NIHF remains unknown in $15-25 \%$ of these cases. Though NIHF has got poor prognosis, several etiologies can be treated with potentially good results. One very rare but treatable condition is Meconium peritonitis (MP). MP was first discovered by Morgagni in 1761, but the first corrective surgery was executed successfully in 1943 by Agerty [3]. MP is a rare fatal disease characterized by sterile inflammatory reaction, secondary to extravasation of meconium into the peritoneal cavity. The pathological event described for the occurrence of MP is intrauterine bowel perforation that may occur during antenatal or postnatal period [4]. The key element for the management consists of prenatal diagnosis and excluding chromosomal disorders, congenital infections and cystic fibrosis. Early surgical procedures to reduce systemic and abdominal inflammation just after birth may improve the outcome of severe MP cases. Recently, the survival rate for MP increased to over $90 \%$. This improvement is the result of an advance in fetal diagnostic techniques, timely intervention and intensive care after birth [5].

\section{Case Report}

A 32 year, G5P2L1D1(IUD)A2, non-consanguineous marriage, was referred to our hospital at 33 weeks 2 days of gestation in view of isolated fetal ascites, diagnosed on antenatal scan at 32 weeks. Mother's blood group was " $A$ " positive, ruling out the possibility of incompatibility. First trimester aneuploidy screen was indicative of low risk. Second trimester serum screening (quad test) and targeted imaging for fetal anomalies were normal. Immuno-hematological work up including indirect coombs test, irregular antibody screening by 3 cell panel was negative. There was no history of congenital anomalies in family or in her previous babies. Work up done to rule out other possible causes including syphilis, cytomegalovirus (CMV), parvovirus B19 and toxoplasmosis, and the reports were normal. Fetal echo was done, which showed structurally normal heart with mild pericardial effusion and echogenic foci in both ventricles. one week later, repeat ultrasound was done which showed moderate fetal ascites, fluid collection in infra diaphragmatic space with echogenic bowel loops floating within it, also few areas of calcification in the bowel loops with prominent Inferior vena cava (IVC), and associated polyhydramnios was noted. Doppler middle cerebral artery peak systolic velocity was $<1.5$ multiples of median which rules out the probability of fetal anaemia (Figure 1). There was no evidence of hydrocephalus, hydrothorax or skin edema. The probable diagnosis of MP was made. Neonatologist and pediatric surgeon's opinion were taken regarding fetal prognosis and further management. She was planned for conservative management with an aim to prolong the pregnancy till 37 weeks. Dexamethasone was given for fetal lung maturation. Subsequently, she went into spontaneous labour and a preterm hydropic female baby of birth weight $3.05 \mathrm{~kg}$ was delivered at 35 weeks, cried immediately after birth, the baby had ascites, and vulval edema (Figure 2). Immediate intubation was done in view of respiratory distress and shifted to neonatal unit for further workup and ventilator support. Placental examination revealed large placenta weighing $1.1 \mathrm{~kg}$, however no other placental abnormality seen (Figure 3). Placental tissue was sent for histopathological examination, Polymerase chain reaction (PCR) for Treponema pallidum, CMV and parvovirus DNA, which all reported negative. Umbilical cord blood sent for cytogenetic analysis and revealed normal chromosomal complement. Post natal ultrasound of the neonate was done which showed gross thick particulated ascites, a giant cyst compressing the IVC, and minimal bilateral pleural effusion. X-ray whole abdomen revealed few spots of intra-abdominal calcification. Emergency exploratory laparotomy was performed on day three of life. Intra-operative findings consisted of clumps of collapsed small bowel loop, a giant meconium cyst of $8 \times$ $10 \mathrm{~cm}$ containing approximately $150 \mathrm{ml}$ of thick meconium, which was drained out, there was also terminal ileal perforation of about $10-14 \mathrm{~cm}$ proximal to ileo cecal junction which was brought out as loop stoma (double barrel ileostomy). Postoperatively baby was extubated and was maintaining saturation with oxygen by nasal prongs, edema started resolving and over all neonatal condition improved (Figure 4). On postoperative day five, baby had one episode of febrile seizure and was started on injection levetiracetum. lleostomy started 
functioning. Low volume enteral feeds were initiated with gradual advancement by seven days of life. lleostomy closure was planned at fifth month follow up. From Third week of life, baby's condition started deteriorating with recurrent episodes of fever and poor weight gain. Higher antibiotics were started, unfortunately the baby could not be saved and succumb to sepsis on fourth week of life.

\section{Discussion}

The occurrence of immune hydrops has been re- duced drastically due to universal use of immunoprophylaxis for red cell isoimmunization. Consequently, NIHF accounts for almost $90 \%$ of cases of HF. MP has been reported as one of the curable etiologies of NIHF. With the evolution in the imaging technologies increasing number of fetuses with MP are being diagnosed prenatally.

Diagnosis of MP is rare before 20 weeks' because peristalsis rarely commences before this time. The median gestational age at initial diagnosis of MP was 24 weeks [6]. According to the study by Uchida $k$, et al.

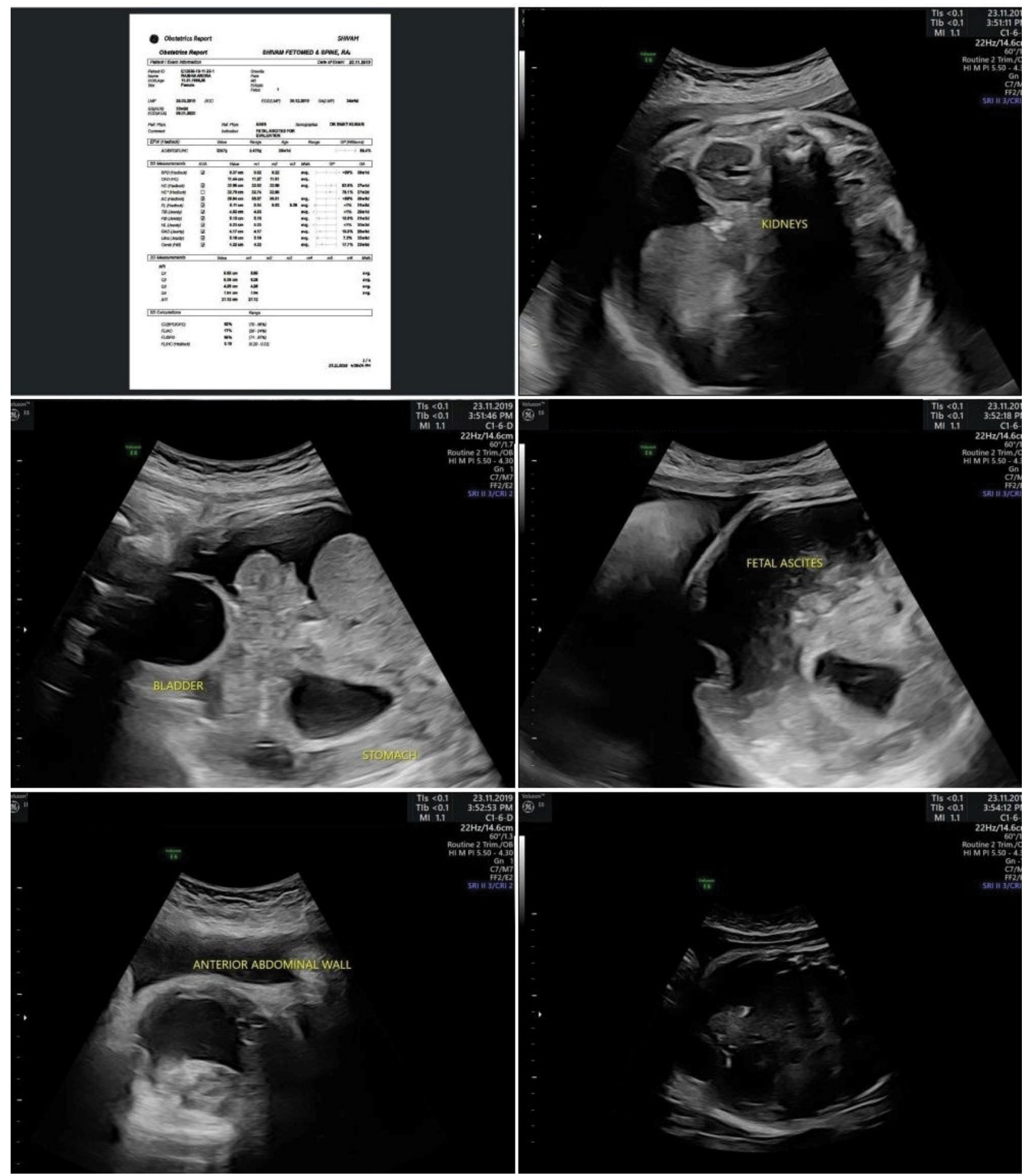




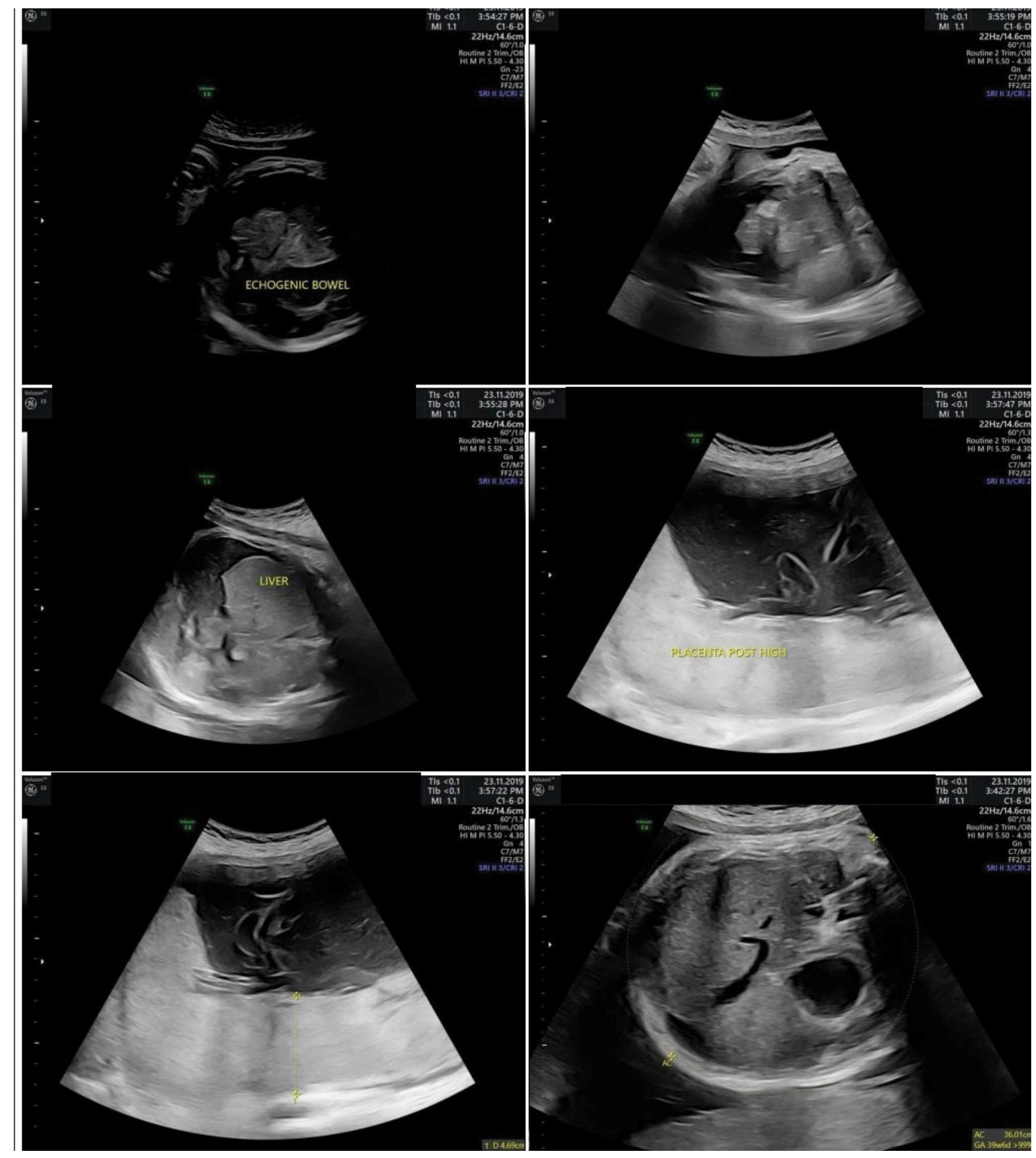



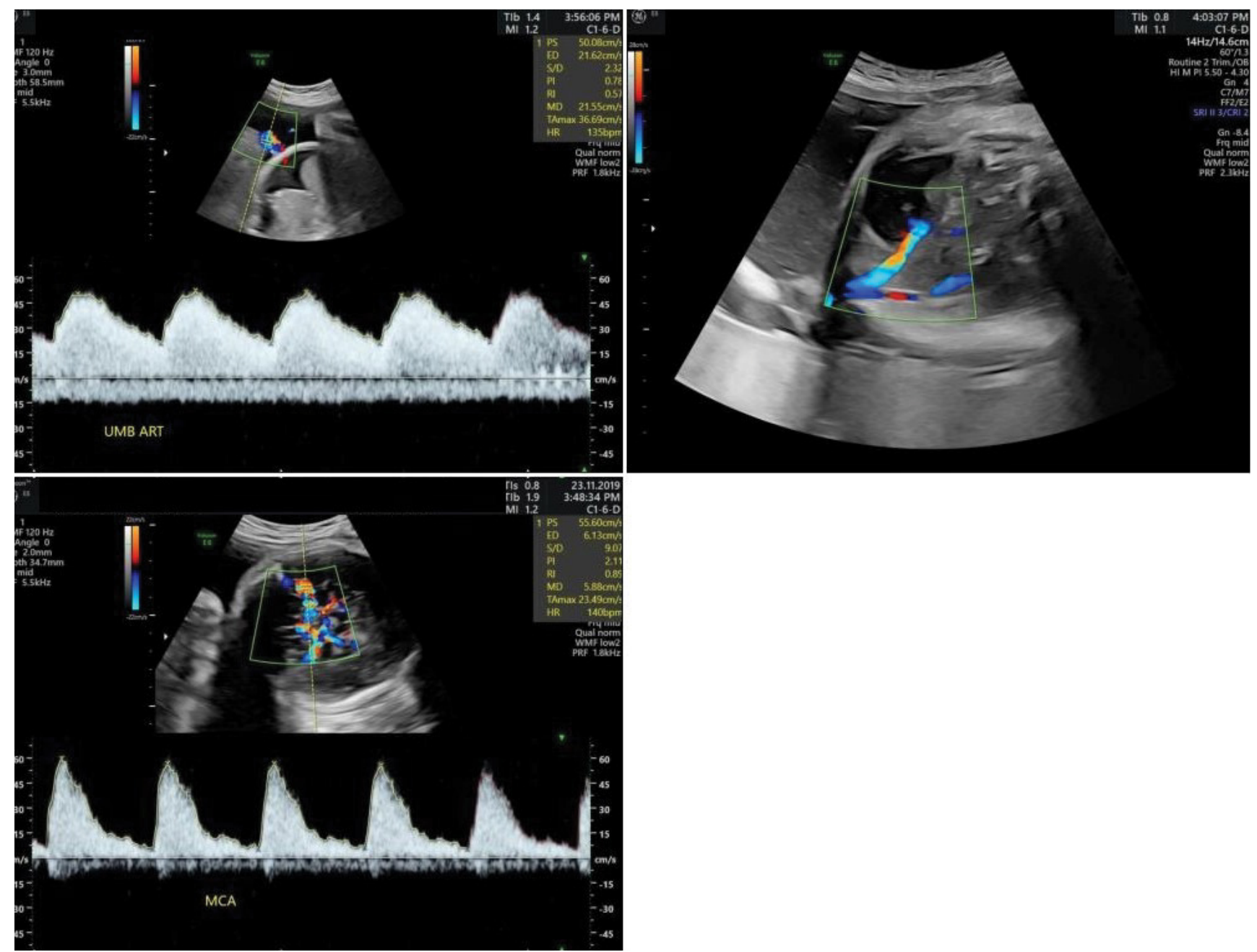

Figure 1: Antenatal ultrasound showing Fetal ascites, Echogenic bowel loops, Polyhydramnios and Normal doppler middle cerebral artery peak systolic velocity.

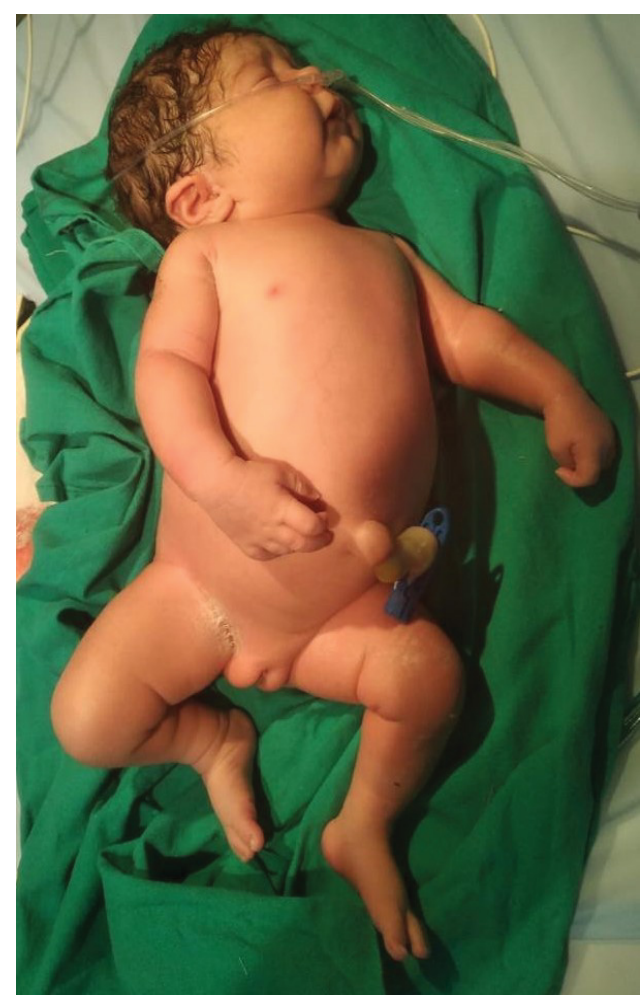

Figure 2: Immediate post-natal period-picture of Hydropic female baby.

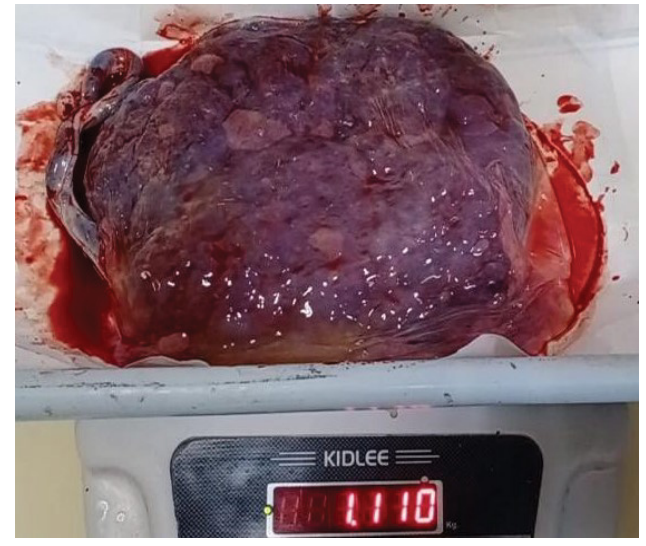

Figure 3: Large placenta.

prenatal diagnosis was made in $73 \%$ of patients. The UItrasound (US) findings with suspected MP were polyhydramnios (100\%), bowel dilatation (53\%), ascites (33\%), and pseudocyst (13\%) [5]. In another study by Ping LM, et al. Fetal ascites (93.3\%) was the most common prenatal US finding [6]. Antenatal US has high specificity (100\%) but low sensitivity (22.2\%) in detecting meconium pseudocyst. Prenatal Magnetic Resonance imaging can improve the low diagnostic yield of prenatal US scan 


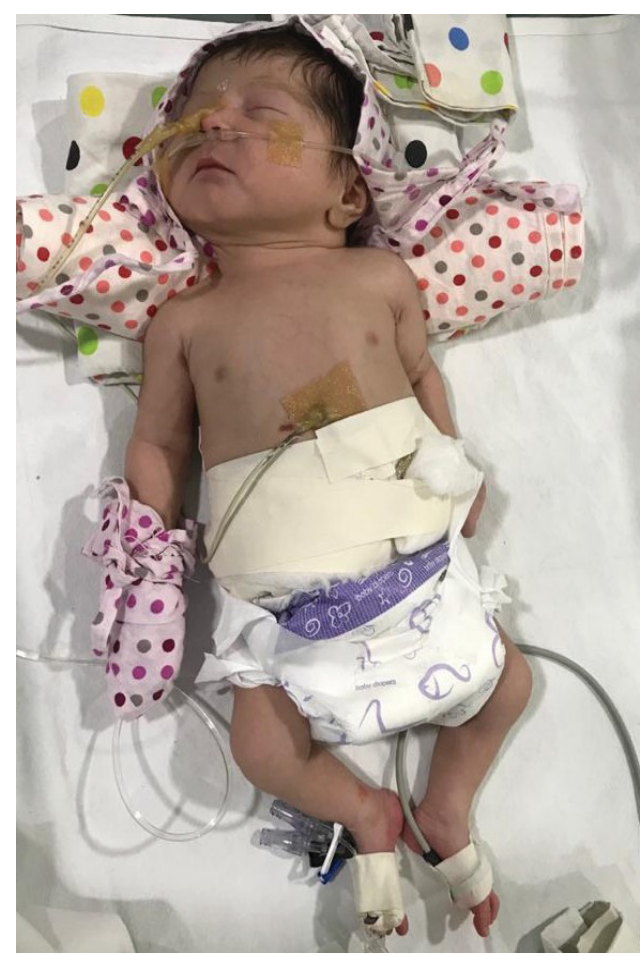

Figure 4: Post-operative picture of baby with overall improved general condition.

for MP. Infants usually present with tense abdominal distension, edematous wall with shiny skin and visible veins, respiratory distress, bilious aspirates/vomiting, failure to pass meconium and features of peritonitis [3]. Abdominal plain X-ray reveals the calcifications in the peritoneal cavity occasionally; the calcifications may extend to the scrotum. On US, MP produces multiple discrete, very highly reflective foci, with acoustic shadowing or occasionally diffuse peritoneal reflectivity (referred to as a 'snowstorm' appearance). Postnatal contrast Computed Tomography (CT) scan is required to define persistent intestinal perforation invisible with prenatal US scan. In most cases surgical intervention is required immediately after birth; Spontaneous healing is reported in rare cases. Caro-Dominguez, et al. reported that postnatal imaging findings that are predictive of the need for surgery include intestinal obstruction, ascites, pneumoperitoneum, and volvulus; however, the presence or distribution of peritoneal calcification was not predictive of the need for surgery [7]. Neonatal sepsis is reported by several authors to be one of the most common causes of mortality as was in our case also.

The incidence of chromosomal abnormalities and genetic syndromes is not increased in cases with MP however a relatively strong association with cystic fibrosis is seen in between $8 \%-40 \%$ of patients [8]. Therefore amniocentesis \& DNA studies for cystic fibrosis should be done if both parents are carriers. However we could not evaluate in our case for this due to non affordability issues.

Meconium is a composite mixture of bile salts, cell debris, and proteins. Spillage of these constituents sec- ondary to in utero bowel perforation has been shown to activate immune cells including macrophages. Infiltration of macrophages into the peritoneum will lead to activation of cellular functions, including phagocytosis, liberation of chemical mediators, and antibody-dependent cell-mediated cytotoxicity. The intense inflammatory reaction leads to the formation of a dense, adherent membrane that practically seals off the intestine at the site of perforation. However, if the sealing is incomplete, a thick-walled cystic space is formed, and meconium will continuously keep collecting in this cystic pocket. Any cause of small bowel ischemia or associated mechanical obstruction such as intestinal atresia, volvulus, intussusception, congenital bands, and meconium plug syndrome, as in cystic fibrosis, may result in the genesis of meconium peritonitis [5].

Depending on the degree of inflammation, three pathological variant of MP can be seen: Fibro-adhesive, cystic and generalized. The most common variant is fibro-adhesive type, resulting from enormous fibroblastic reaction; cystic type is seen when the perforation site is not completely sealed and thus forming a thickwalled cyst. Generalized MP presents as diffuse bowel thickening of the affected segment, peritoneal fibrosis and calcium deposits. Recent studies do not provide clear guidelines concerning surgical strategies for MP. Enterostomy, T-tube ileostomy, primary anastomosis, Bishop-koop, santulli and Mikulicz are common procedures for MP. Although the type of surgical procedure seems to depend upon clinical manifestation, general condition of the patient and surgeon's preferential technique, few comparative studies have been performed. Miyake, et al. consider primary anastomosis as safe option for almost all patients with MP except for those with very low birth weight and in an unstable condition. The advantages of primary anastomosis are reduced hospital stay, avoidance of stoma related morbidity and second laparotomy for stoma closure. $\mathrm{Nam}$, et al. reported a preference for primary resection and anastomosis of the intestinal segment involved. However severe complications related to the surgical procedure itself, such as peritonitis from anastomotic leakage and perforation caused by frequent manipulation are more often seen in primary anastomosis. It is difficult to assess objectively the viability and condition of the intestine, therefore karimi, et al. recommended resection with temporary double barreled enterostomy as the safest treatment [9]. The surgical strategy for our case was two stage approach with abdominal drainage or temporary enterostomy and elective reconstruction of intestinal continuity (stoma closure) at fifth month of life. However stoma closure could not be done in our case as the baby succumbed on fourth week of life due to sepsis. Recently, the first choice for cystic type MP was abdominal decompression by catheterization closed drainage. 
Table 1: Zangheri's grading system for meconium peritonitis.

\begin{tabular}{|l|l|l|}
\hline \multicolumn{2}{|l|}{ Score } & \multicolumn{2}{l|}{} \\
\hline 0 & & Intra-abdominal calcification \\
\hline 1 & A & Intra-abdominal calcification with ascites \\
\hline & B & Intra-abdominal calcification with pseudocyst \\
\hline 2 & C & Intra-abdominal calcification with bowel dilation \\
\hline 3 & & $\begin{array}{l}\text { Intra-abdominal calcification with any two of the } \\
\text { previous findings }\end{array}$ \\
\hline $\begin{array}{l}\text { Intra-abdominal calcification with all of the } \\
\text { previous findings }\end{array}$ \\
\hline
\end{tabular}

In previous reports of MP presenting as hydrops, all cases were of cystic type [10]. The likely cause of hydrops in MP can be due to compression of inferior vena cava by giant cyst, compromising the venous return, thereby increasing umbilical venous pressure. A similar mechanism may explain hydrops in our case.

Assessment of patients is done by Zangheri's grading system for MP (Table 1). In isolated cases of calcification (score 0 ), the probability for surgery is minimal. In other patients with score 1 to 3 , the probability increases to over $50 \%$ [3].

Prognosis and natural evolution of meconium peritonitis, when it is diagnosed in the fetal period, are different from that diagnosed postnatally. If ultrasound during prenatal period is suggestive of only intraperitoneal calcifications (also known as simple MP) without bowel dilatation, polyhydramnios, ascites or pseudocyst, the prognosis is favorable. If all the findings listed above are associated, the prognosis is bad and need for surgical intervention increases [9]. Uchida K, et al. also verified that early surgery is an effective way to reduce intra-abdominal and systemic inflammation, which helps to enhance the outcome of severely affected patients [11].

\section{Conclusion}

Prenatal diagnosis is crucial for the first step of perinatal therapy for MP. Management and the need for surgery depend on the clinical presentation and the overall condition and gestational age at birth of the newborn. Surgery is required when signs of intestinal obstruction are present. Early diagnosis, use of higher antibiotics and active management of acid base imbalance, superimposed bacterial peritonitis, and septic shock can prevent mortality. Timing of delivery should rely on composite decision of gynecologists, neonatologists, and neonatal pediatric surgeons in perinatal and maternal care centers. Surgery performed within 24 hours in newborns with bowel obstruction may also improve their outcome.

\section{Declarations}

\section{Ethical approval and consent to participate}

Not applicable.

\section{Informed consent}

Informed consent was obtained from the patient.

\section{Conflict of interest}

The authors declare that they have no competing interests.

\section{Funding}

None.

\section{Acknowledgements}

Not applicable.

\section{References}

1. Meng D, Li Q, Hu X, Lifang W, Shuyin T, et al. (2019) Etiology and outcome of non-immune hydrops fetalis in southern china: Report of 1004 cases. Sci Rep 9: 10726.

2. Kosinski $P$, Krajewski $P$, Wielgos $M$, Jezela-Stanek $A$ (2020) Nonimmune hydrops fetalis-prenatal diagnosis, genetic investigation, outcomes and literature review. J Clin Med 9: 1789.

3. Gaddam S A, Tirunagari S (2018) Meconium peritonitis - A rare cause of ascites. JCR 8: 190-193.

4. Turkyilmaz G, Kurt D, Sarac Sivrikoz T, Kalelioglu I, Has R, et al. (2019) Prenatal diagnosis and conservative management of complex meconium peritonitis: A case report. J Clin Invest Surg 4: 48-52.

5. Uchida K, Koike Y, Matsushita K, Nagano Y, Hashimoto K, et al. (2015) Meconium peritonitis: Prenatal diagnosis of a rare entity and postnatal management. Intractable Rare Dis Res 4: 93-97.

6. Ping LM, Rajadurai VS, Saffari SE, Chandran S (2017) Meconium peritonitis: Correlation of antenatal diagnosis and postnatal outcome - an institutional experience over 10 years. Fetal Diagn Ther 42: 57-62.

7. Caro-Dominguez $P$, Zani A, Chitayat D, Alan Daneman (2018) Meconium peritonitis: The role of postnatal radiographic and sonographic findings in predicting the need for surgery. Pediatr Radiol 48: 1755-1762.

8. Vlok SSC, Du Plessis V (2015) Meconium pseudocyst associated with congenital CMV infection. S Afr J Rad 2.

9. Tembely Samba, Kouame Yapo Guy Serge, Yaokreh Jean Baptiste, Odehouri-Koudou Thierry Herve, Thomas HA, et al. (2019) Meconium peritonitis: Management in a developing country in sub-sahara africa. EC Paediatrics.

10. Rajendran UD, Govindarajan J, Balakrishnan U, Chandrasekaran A, Amboiram P (2019) Meconium peritonitis: A rare treatable cause of non-immune hydrops. Pediatr Gastroenterol Hepatol Nutr 22: 576-580.

11. Jiang Y, Pan W, Wu W, Weipeng W, Suna S, et al. (2019) Can early surgery improve the outcome of patients with meconium peritonitis? A single-center experience over 16 years. BMC Pediatr 19: 473. 